\title{
Perspectives on Placebo: The Psychology of Neurofeedback
}

\author{
Kirtley E. Thornton * \\ Neuroscience Center, Charlotte, North Carolina, USA
}

\begin{abstract}
The application of operant conditioning with EEG variables to produce changes in behavior has been gaining increasing interest in research and application areas. However, the methodology has come under scrutiny and criticism for its potential placebo effects. This article will examine those issues from the traditional methodologies of demonstrating effectiveness (control group, sham treatments) as well as examine the possible biochemical and electrophysiological effects of a placebo response. Specifically, the role of endorphins and dopamine and their relationship to the alpha and beta frequency in the placebo response will be examined. The research addressing the diffusion tensor imaging (DTI) and functional magnetic resonance imaging (fMRI) correlates of the intervention will be examined.

Keywords: neurotherapy; EEG biofeedback; placebo; alpha; endorphins; dopamine

Citation: Thornton, K. E. (2018). Perspectives on placebo: The psychology of neurofeedback. NeuroRegulation, 5(4), 137-149. http://dx.doi.org/10.15540/nr.5.4.137

*Address correspondence to: Kirtley Thornton, PhD, 9635

Southern Pine Blvd., Suite 105, Charlotte, NC 28273-5558, USA.

Email: ket@chp-neurotherapy.com

Copyright: (c) 2018. Thornton. This is an Open Access article distributed under the terms of the Creative Commons Attribution License (CC-BY).

\author{
Edited by: \\ Rex L. Cannon, PhD, Knoxville Neurofeedback Group, Knoxville,

\section{Reviewed by:} \\ Rex L. Cannon, PhD, Knoxville Neurofeedback Group, Knoxville, \\ Tennessee, USA \\ Randall Lyle, PhD, Mount Mercy University, Cedar Rapids, lowa, \\ USA
} Tennessee, USA
\end{abstract}

\section{Definitions of Placebo and Effects}

The common thread of varying definitions is that a placebo has (a) a positive effect on the patient's perception and self-report; and (b) does not have any physical beneficial effects on the illness for which it is prescribed.

Thibault and Raz (2017) conclude that "EEG-nf works, but it likely relies heavily on placebo phenomena" (p. 683). They further argue that "mental health professionals stand to benefit from studying the ubiquitous placebo influences that likely drive these treatment outcomes" (p. 679). "EEG-nf studies largely neglect investigating treatment mechanisms that rely on participant motivation, belief in the treatment administered, interacting with a practitioner, level of positive feedback, and sense of control of their brain signal" (p. 684). This paper will investigate the logic and evidence regarding the potential placebo effect in the neurotherapy (NT) situation.
In order to demonstrate a placebo effect, a research project must meet the following criteria document: (a) the expectation of the subject or study characteristics which encourage such an expectation; (b) the concomitant biological correlates (neurochemical, EEG, fMRI, DTI correlates of the expectation); and (c) the lack of a relationship between the biological correlates of the placebo and the biological correlates of the illness in question. To document that the effect is a real, substantive effect, (d) the results must distinguish between the effects of the intervention which have no measurable physical effect on the problem in question (a placebo) and those interventions which have a direct measurable physical effect on the problem.

It is clear that there is a real physiological effect from a placebo. The mechanism is not the pill or the treatment but rather the subject's perception that there is a value (positive/negative) in the pill or treatment. It is this belief that creates a change in the physiology of the brain and thus underlies the 
perceived changes in symptoms. It is the goal of this paper to understand the relationship between the perception of change and the underlying electrophysiology and biochemistry of that change. We will address what is known/unknown about this relationship. If NT is a placebo response, then it is necessary to document the physiology of placebo response and its relationship to reported changes obtained with NT.

There is some inherent ambiguity built into the question of whether NT is a placebo in the traditional sense. It should be noted that the placebo argument originated in drug research addressing physical conditions, thus a physical intervention (pill, etc.) addressing a physical condition. NT is not a pill addressing a physical condition but an operant conditioning method to change the EEG signal which is related to behavior (such as attention). In both situations, however, the concern is whether the subject's subjective response is influencing the results which are tied to the relevant behavior issue (attention, etc.) and how much. In the case of attention-deficit disorder (ADD), if the "illness" is excessive theta activity and deficient beta and a placebo or NT changes the theta and beta values and relevant behavior, then the question is: Has a "cure" occurred? It is not assumed that the theta/beta issues are the sole physical issue in the attentiondeficit/hyperactivity disorder (ADHD) subject.

This paper will examine research on the physical/physiological effects related to the placebo response (endorphins, dopamine, EEG signal) and determine if it is credible to account for the NT effects as a placebo.

\section{Placebos work on patient's perceptions}

Hróbjartsson and Gøtzsche (2010) reviewed the literature on placebos for 60 clinical conditions and concluded that there was a placebo effect in the clinical conditions of pain, nausea, asthma, and phobia but no effect in smoking, dementia, depression, obesity, hypertension, insomnia, and anxiety. Thus, only $7 \%$ of the conditions studied had a documented placebo effect. The perception of pain condition appears to be most responsive to the placebo effect (approximately 30 to 60 percent of people report a positive effect; Cherry, 2018).

In addition, not all subjects respond to the placebo effect. Beecher (1955) reviewed 26 studies and found on average $32 \%$ of the patients responded to placebo. In addition, it appears that some subjects are not responsive to the placebo effect due to genetics. "Predisposition to respond to placebo treatment may be in part a stable heritable trait" (Hall, Loscalzo, \& Kaptchuk, 2015, p. 20).

Rossi (1986) concluded that "placebo is about 55$60 \%$ as effective as active medications irrespective of the potency of these active medications." Rossi also mentions that "in a study of morphine, there was a $50 \%$ pain reduction in $75 \%$ of the patients treated. The placebo group had a $50 \%$ pain reduction in $36 \%$ of the patients" (WRF, n.d.)

Kaptchuk et al. (2010) studied two groups of irritable bowel syndrome (IBS) patients. One group did not receive any treatment, while the other group was provided with pills labeled placebo and were told they were "fake, inert drugs" and that the "placebo pills, something like sugar pills, have been shown in rigorous clinical testing to produce significant mindbody self-healing processes" (Kaptchuk et al., 2010). The group receiving the fake placebo drugs reported twice as much symptom relief as the no treatment group and comparable to the best real medicine for IBS. According to Feinberg (2013), Kaptchuk's interest is "not if, but how, placebo effects work," as Kaptchuk argues that "sham treatment won't shrink tumors or cure viruses" (p. 36).

Wechsler, Kelley, and Kaptchuk (2011) studied 40 asthma patients with four different interventions. They found that only the real treatment showed results. However, there was no difference between the patient's subjective response to the real versus sham treatments. The patient's subjective response was not consistent with the objective measures. Thus, the placebo does not cure the problem but does result in a patient's perception of change.

The argument that NT is solely a placebo effect for all of the participants who have been involved in the treatment must be understood in the context that the placebo effect has only been documented in $7 \%$ of the conditions, by $32 \%$ of the patients, is determined, in part, by genetics and is about half as effective as morphine (in terms of patient response rates). Thus, the "ubiquitous placebo influences" are not found everywhere.

\section{Placebo's Physical Effects on the Body and Brain}

The placebo's physical effects on activity in brain structures has been documented in Parkinson disease (Benedetti et al., 2004; de la FuenteFernández et al., 2001), pain (Eippert et al., 2009; 
Petrovic, Kalso, Petersson, \& Ingvar, 2002; Wager et al., 2004; Zubieta et al., 2005), depression (Leuchter, Cook, Witte, Morgan, \& Abrams, 2002), and anxiety (Furmark et al., 2008; Petrovic et al., 2005). The anxiety and depression results are in contradiction to the Hróbjartsson and Gøtzsche Cochrane review. The hypothesis resulting from these studies suggest a prefrontal control mechanism that effects activity and neurotransmitters. A full understanding of the structural, functional, biochemical, and electrophysiological effects is required.

"Researchers have found that placebo treatmentsinterventions with no active drug ingredients-can stimulate real physiological responses, from changes in heart rate and blood pressure to chemical activity in the brain, in cases involving pain, depression, anxiety, fatigue, and even some symptoms of Parkinson's" (Feinberg, 2013).

\section{Endorphins}

Levine, Gordon, Jones, and Fields (1978) first reported that blocking the release of endorphins (chemically) by opioid antagonist naloxone would stop the placebo effect. Endorphins are the brain's natural pain relievers and are similar to morphine and opiates.

Benedetti et al. (2004) concluded that "there is ample evidence that expectancy-based placebo effects are mediated by endogenous opioids." Levine et al. (1978) noted that naloxone blocks the brain's ability to soak up endorphins. Volavka, James, Reker, and Cho (1979) showed that "naloxone elicited a significant slowing of the average alpha frequency" (p. 1267). This slowing effect would most likely be manifested in the lowering of the peak frequency of alpha. Thus, the alpha frequency appears to be involved in the placebo/endorphin response. The interrelationships between different measures of the alpha frequency (magnivolts, relative power, peak frequency) pose a complex problem, which is beyond the scope of this paper.

Lipman et al. (1990) reported that, in chronic pain subjects, the placebo responders had higher concentrations of endorphins in the cerebrospinal fluid than the placebo non-responders. Wager, Scott, and Zubieta (2007) concluded that placebo effects involve opioid activation in opioid receptor rich regions including "periaqueductal gray and nearby dorsal raphe and nucleus cuneiformis, amygdala, orbitofrontal cortex, insula, rostral anterior cingulate, and lateral prefrontal cortex" (p. 11056). Thus, the placebo effects involve a similar activity or results suggest that endogenous opioid release in core affective "brain regions is an integral part of the mechanism whereby expectancies regulate affective and nociceptive circuits" (p. 11056).

However, it has also been reported that the placebo effect can occur without the involvement of opioids and naloxone can only partially inhibit the placebo analgesia effect in some situations (Amanzio \& Benedetti, 1999; Gracely, Dubner, Wolskee, \& Deeter, 1983; Grevert, Albert, \& Goldstein, 1983).

The relationship between endorphins and the EEG has not been studied directly. However, there is indirect evidence that the endorphins increase the level of alpha magnitudes. Mimasa et al. (1996) "found that the larger the changes in beta-endorphin following exercise, the higher the appearance rate of the alpha wave in EEG. There was a positive and significant correlation $(r=.563, p<.05)$ between the increase in alpha wave component and that of the plasma beta-endorphin." It is unclear whether the increase was a magnitude or relative power increase. Thornton (2016) reported a positive correlation $(r$ $=.66, p<.05$ ) between magnitude and relative power measures of alpha. However, Crabbe and Dishman (2004) were able to document that exercise results in increased alpha magnitudes. Pfefferbaum et al. (1979) found that "beta-endorphin and morphine produced similar increases in alpha power within 5 to 15 minutes after injection."

Peniston and Kulkosky (1989) compared a nonalcoholic control group, a traditionally treated alcoholic control group, and alcoholics receiving brainwave training (BWT). The BWT group showed significant increases in percentages of EEG record in alpha and theta rhythms (the focus of the intervention), increased alpha rhythm amplitudes, and improved outcome compared to the other two groups.

However, the alcoholics receiving abstinence, group psychotherapy, or antidepressants showed a significant elevation in serum beta-endorphin levels at the conclusion of the experiment. "This neuropeptide is an index of stress and a stimulant of caloric (e.g., ethanol) intake" (Peniston \& Kulkosky, 1989, p.271). "Beta-endorphins play a role in certain behavioral patterns (stress, alcoholism), in obesity, diabetes, and psychiatric diseases" (Peniston \& Kulkosky, 1989). Dalayeun, Norès, and Bergal (1993) application of brainwave treatment, a relaxation therapy, appears to counteract the increase in circulating beta-endorphin levels seen in the control 
group of alcoholics. This partially explained result stands in contrast to the expected increases in alpha associated with increases in endorphin levels.

It appears that the placebo effect involves endorphins and alpha magnitudes, relative power and peak frequency. Thus, when a subject believes an intervention is going to be successful, the endorphins increase and the magnitudes of alpha increase, as well as probably other frequencies. It remains unclear whether the increase in alpha magnitudes is a result of the placebo effect or NT. Even an increase in the alpha and theta magnitudes during a session or treatment period is not an argument against a placebo effect as the argument could be made that the placebo's effect increases during the session gains or as more sessions are employed.

\section{Dopamine}

De la Fuente-Fernández et al. (2001) found that placebo-induced expectation of motor improvement activates endogenous dopamine in the striatum of Parkinsonian patients. Additional studies have pointed to dopamine involvement in the placebo response in pain conditions (Scott et al., 2008; Zubieta et al., 2005; Zubieta \& Stohler, 2009).

Hall et al. (2012) stated that "Catechol-Omethyltransferase (COMT), an important enzyme in dopamine catabolism, plays a key role in processes associated with the placebo effect such as reward, pain, memory, and learning." As "COMT activity decreased, theoretically making more dopamine available in the prefrontal cortex, placebo responses increased in a linear fashion...associated with a positive outcome only in groups given a placebo (and not in the waitlist control group) is of particular importance, as it indicates that it is a predictor of the placebo effect, not just improvement in general" (Hall et al., 2012).

Dopamine has been shown to be part of the ADHD condition. An increase in psychostimulants should result in increases in dopamine levels and increases in EEG arousal frequencies (alpha and beta) and decreases in lower frequencies (delta and theta). "Methylphenidate: Ritalin produces a decrease in delta and theta, with a more pronounced posterior alpha increase and an increase in low beta, with effects delayed up to 6 hours, compared to the rapid effects of the amphetamines" (Gunkleman, 2009, p. 4). "Medication resulted in normalization of theta power, but, after medication, increased relative beta was also apparent in the female ADHD group" (Clarke, Barry, McCarthy, Selikowitz, \& Johnstone,
2007). This result was also obtained in adults Bresnahan, Barry, Clarke, and Johnstone (2006), who reported that following medication, there was a "significant reduction in slow wave activity in the ADHD adult group to levels similar to those in the control group."

However, these findings have not been without contrary evidence. Lubar, Swartwood, Swartwood, and Timmermann (1996) failed to show increases in qEEG indicators of cortical arousal with methylphenidate. Other researchers (Barkley, 1998; Ernst et al., 1994; Matochik et al., 1994) have failed to demonstrate the neurophysiological effects of Ritalin at the cortical level.

There is solid research in the efficacy of operant conditioning (Staddon \& Cerutti, 2003). The research has documented that NT can increase beta magnitudes and decrease theta magnitudes as well as increase alpha (Lubar et al., 1996; Sherlin, Arns, Lubar, \& Sokhadze, 2010). The effect can be maintained up to 2 years (Gani, Birbaumer, \& Strehl, 2008; Leins et al., 2007) and is maintained after cessation of medication (Monastra, Monastra, \& George, 2002). Endorphins and dopamine have short-term effects. Foley et al. (1979) reported the half-life of beta-endorphin was 37 to 93 minutes. "The half-life of dopamine effect is 2 min" (ADHB, 2018). It is conceptually challenging to understand how it is possible that the placebo effect could be manifested 2 years later.

In conclusion, activation of an endorphin response appears tied to the alpha frequency, while dopamine's effects are predominantly in the beta frequency. NT and medications appear to break apart the naturally positive relationships between the magnitudes of the theta, alpha, and beta frequencies.

\section{Neurotherapy and Structural/Functional Changes on fMRI, MRI, and DTI}

Lévesque, Beauregard, and Mensour (2006) studied twenty ADHD unmedicated children who were divided between the experimental group (EXP; $N=15)$ and control (CON; $N=5)$ and baseline measure were obtained on two cognitive measures (Digit Span, Integrated Visual and Auditory [IVA] Continuous Performance Test [CPT]) and two questionnaire variables (Conners Parent Rating Scale, inattention and hyperactivity component). Following 40 sessions of NT, inhibiting theta microvolts $(4-7 \mathrm{~Hz})$ and rewarding beta microvolts $(12-15 \mathrm{~Hz}, 15-18 \mathrm{~Hz})$ at $\mathrm{Cz}$, there were significant improvements in the EXP 
group on the two cognitive and two questionnaire variables. In addition, a repeat $\mathrm{fMRI}$ scan was conducted while they (EXP and CON) performed a Counting Stroop task. The initial evaluation indicated significant increases in both groups in the left superior parietal location (Brodmann area 7). Postintervention fMRI scan revealed that the EXP group had increased activation of BA $24 \mathrm{~b}-\mathrm{c}$ and 32 , which involve the anterior cingulate cortex, known to be involved in attentional issues (Bush et al., 1999). The CON group "did not receive an attentional training lasting the same time duration than the NT received by EXP subjects," which the authors suggested needs to be further evaluated (Lévesque et al., (2006). Thus, the NT intervention showed positive behavioral attentional and relevant fMRI results, with some qualifications regarding the $\mathrm{CON}$ group intervention.

In this study, the posttreatment Counting Stroop task requested the subjects to indicate the number of words presented (neutral condition) and then provided with an interference condition, during which the words one, two, three, and four were presented. The analysis compared the interference condition to the neutral condition. For both the EXP and CON groups, there was a significant locus of activation in the left superior parietal lobule (BA 7; fMRI) at both time periods. For the EXP group, at time 2, there were significant activations in the right BA 32 and left the caudate nucleus. Banich et al. (2000) reported that the anterior cingulate cortex is employed in selecting an appropriate response and allocate attentional resources. BA 32 is located in the anterior cingulate cortex. The caudate nucleus is involved in the inhibitory control of action (Nestler, Hyman, \& Malenka, (2009). Thus, NT interventions appear to have an additional and relevant effect on brain structures that were not present for the CON group. Criteria \#4, presented in this article, argues that if a treatment results in relevant change then it is real and not a placebo result. The NT intervention appears to have met that criteria, apart from the CON group intervention qualification.

Ghaziri et al. (2013) examined white matter (WM) and grey matter volume (GMV) in a sample of 30 participants under EXP, sham, and CON group conditions. They employed NT rewarding 15-18 Hz at F4 and P4 for 40 sessions (30 min each). Pre- and posttreatment data were available for the Integrated Visual and Auditory (IVA) test. The EXP and sham treatment showed significant increases on the IVA, while the sham group showed significant increases in the visual attention measure. The CON showed no significant improvements on either measure. The areas of interest for the structural magnetic resonance imaging (MRI) study and the fractional anisotropy (FA) measure of DTI involved the cingulum bundle (CB), superior longitudinal fasciculus (SLF), inferior longitudinal fasciculus (ILF), and the splenium of the corpus callosum (SCC).

For the EXP group, there were significant increases in fractional anisotropy (FA) in the right and left $C B$, right anterior corona radiata and SCC as was as left SLF and ILF. "These WM pathways are known to be associated with sustained attention" (Ghaziri et al., 2013, p. 269).

This result satisfies criteria (d) mentioned at the beginning of this paper which states "must distinguish between the effects of the intervention which have no measurable physical effect on the problem in question (a placebo) and those interventions have a direct measurable physical effect on the problem." In this case, the EXP group had a direct measurable physical effect on the problem of attention, as the affected "WM pathways are known to be associated with sustained attention." Thus, the intervention must be viewed not as a placebo but a valid, relevant intervention on the "cause" of the problem-WM pathways.

The visual attention performance correlated with the FA measures in the left SLF and left ALIC (left anterior limb of the internal capsule). In the sham and CON conditions, there were no significant FA increases at posttesting.

For the EXP group, the GMV measures showed increases in Brodmann areas 9, 20, 19, 6, 47, 22 and 7 while the sham group showed increases in BA 10, 6 , and 18. Thus, the EXP group activated BA areas $9,19,20,22$, and 47 that the sham group did not. The sham group received training "for approximately 20 hours, participants in the SHAM group had to undergo a perceptual-cognitive 'training', consisting of staring at the computer screen and staying focused with respect to the animation displayed on that screen. The members of this group also received hours of personal coaching to pay attention visually" (Ghaziri et al., 2013, p. 269). This coaching, perhaps, explains their improvement on the visual attention IVA. Thus, the sham group did not result in increased communication patterns (increases in FA values) but did result in increased GMV in frontal areas (BA areas 10 and 6) while the EXP group increased frontal GMV in BA 9 and 47 . The study was the "first empirical demonstration that NT can lead to microstructural changes in white and gray matter" (Ghaziri et al., 
2013). This study had the sham group engaged in training which addressed the problem in the Lévesque et al. (2006) study with the control group.

The lack of significant FA increases in the sham condition presents a serious challenge to the NT as placebo argument. This result indicates that the NT intervention is resulting in a substantive change in the physical functioning (FA) of the brain while the sham intervention does not. This is evident in both the communication measure (FA) and different GMV increases, reflecting that the NT approach is fundamentally changing. The presence of a sham condition argues definitively against a placebo effect.

Gevensleben, Holl, Albrecht, Schlamp, et al. (2009) and Gevensleben, Holl, Albrecht, Vogel, et al. (2009) studied 94 ADHD children with a randomization approach involving multicenters and a sham condition (a computerized attention skills training). The NT intervention involved decreasing theta $(4-8 \mathrm{~Hz})$ and increasing beta $(13-20 \mathrm{~Hz})$. The post-qEEG data showed reduced theta power, demonstrating efficacy and specificity. Gevensleben et al. (2010) conducted a 6-month follow-up on the 2009 study and found that the improvements in the NT group were comparable to the effects at the end of the training period (effect size of .71), employing parent rating scales. Thus, the maintenance of effect at the 6-month time period is problematic for a placebo explanation in addition to the reduction of theta magnitudes which is not concurrent with a reduction in beta magnitudes, the naturally occurring pattern.

\section{Clinical Conditions}

We will examine the different clinical conditions to determine if the NT effects can justifiably be called a placebo effect.

\section{ADD}

The ADD condition has been the subject of many investigations with modern medical imaging technology. Initial research focused on the theta/beta values. Eyes-closed, resting EEG data indicated that higher relative power of theta and reduced relative power of alpha and beta, as well as elevated theta/alpha and theta/beta ratios being associated with ADD/ADHD (Barry, Clarke, \& Johnstone, 2003). There are other studies supporting this pattern (Chabot \& Serfontein, 1996; Matsuura et al.,1993). Several EEG research reports have shown that beta activity is related to sustained attention (Arruda, Zhang, Amoss, Coburn, \& Aue, 2009; Molteni,
Bianchi, Butti, Reni, \& Zucca, 2007), thus relevant to the ADHD's problem in attention.

NT approaches (combined theta/beta training with the training of slow cortical potentials, SCPs) obtained a reduction of theta activity (Gevensleben et al., 2010). Improvements on the EEG measures was associated with improvements on an ADHD rating scale. Similarly, Monastra et al. (2002) reported a decrease of the theta/beta quotient in a group of children with ADHD with an initially enhanced theta/beta quotient. The clinical value of the theta/beta ratio resides in its high correlation $(r=.99)$ and age-related changes in the ADHD behavioral symptomatology (Snyder \& Hall, 2006).

More recently, the research has focused on deficits in white matter tracts (Hamilton et al., 2008; Konrad et al., 2012; Niogi, Mukherjee, Ghajar, \& McCandliss, 2010; Pavuluri et al., 2009; Qiu et al., 2011). Niogi, Mukherjee, Ghajar, and McCandliss (2010) conducted a DTI study (healthy participants) and showed a positive correlation between FA values in the anterior limb of the internal capsule and performance on a sustained attention task.

Kong et al. (2006) has reported DTI research on ADHD which reported significantly lower FA values in the left hemisphere involving the frontoparietal networks (SLF, ILF, and CB), or are implicated in interhemispheric processing within parietal areas ( SCC). Van Ewijk, Heslenfeld, Zwiers, Buitelaar, and Oosterlaan (2012) conducted a meta review of the DTI area and ADHD and concluded that "alterations in white matter integrity were found in widespread areas, most consistently in right anterior corona radiata, right forceps minor, bilateral internal capsule, and left cerebellum."

Thus, the ADHD pattern is one of elevated theta, reduced beta activity, and decreased FA values. The decreased FA values would imply lowered coherence and phase values. Treatment approaches have indicated the ability of NT to decrease theta and increase beta levels. Specific interventions on coherence and phase values in the ADHD subject has not been published to the best of the author's knowledge.

If the placebo effect of alpha magnitude increase was occurring during the NT intervention, one would expect an increase in theta and beta1 magnitudes and thus no significant change in the theta/beta ratios. Yet the research (Gevensleben, Holl, Albrecht, Schlamp, et al., 2009; Monastra et al., 2002) 
report decreases in theta magnitudes and changes in theta/beta ratios which employ magnitude values.

\section{Drug abuse}

Peniston and Kulkosky (1989) studied alcoholics receiving BWT and showed a gradual increase in alpha and theta brain "rhythms" across the 15 experimental sessions. It is assumed that rhythms means amplitudes. The reason for the intervention was the previous research which hypothesized a decreased alpha level in alcoholics (Gabrielli et al., 1982). Saletu, Anderer, Saletu-Zyhlarz, Arnold, and Pascual-Marqui (2002) documented with qEEG and LORETA mapping studies of detoxified alcoholdependent patients, as compared with normal controls, higher values of absolute and relative beta power, and lower values in alpha and delta/theta power for the alcohol patients.

Sokhadze, Cannon, and Trudeau (2008) reviewed the research on substance use disorders and biofeedback and concluded that "alpha theta training - either alone for alcoholism or in combination with beta training for stimulant and mixed substance abuse and combined with residential treatment programs, is probably efficacious.... Based on the guidelines jointly established by the Association for Applied Psychophysiology and Biofeedback (AAPB) and the International Society for Neurofeedback and Research (ISNR)" (p. 1). The NT intervention used most frequently was the rewarding of alpha $(8-13 \mathrm{~Hz})$ and theta $(4-8 \mathrm{~Hz})$ in eyes-closed condition. The Scott, Kaiser, Othmer, and Sideroff (2005) modification involved initially rewarding C3FPZ and C4-PZ SMR (12-15 Hz) and beta (15-18 $\mathrm{Hz}$ ) while inhibiting theta $(2-7 \mathrm{~Hz})$ and high beta (22$30 \mathrm{~Hz}$ ), and then followed up with the Peniston protocol (reward alpha and theta). Abstinence was obtained for $77 \%$ of the EXP subjects and $44 \%$ for the controls at 1 year after intervention. The controls were involved in the Minnesota Model 12-steporiented program as well as additional time in treatment which equaled the EXP time in treatment.

There remains the possibility of a placebo effect with endorphins and dopamine affecting the alpha and beta frequencies. However, the endorphins and dopamine would be increasing alpha and beta. The authors did not report the changes in the frequencies. However, the results were better than most rehabilitation programs and the 1-year time reassessment is a long time to expect a placebo effect to last.

\section{Depression}

There are numerous studies that report decreases in depression on the Minnesota Multiphasic Personality Inventory (MMPI) and other measures following NT, typically involving alpha/theta protocols (Cheon, Koo, \& Choi, 2016; Grin-Yatsenko et al., 2018; Hammond, 2005; Raymond, Varney, Parkinson, \& Gruzelier, 2005; Wang et al., 2016). Scott et al. (2005) reported improvements on the MMPI which included the experimental group's changes and exhibited a significant improvement compared with the changes in the control subjects on the Hypochondriasis, Depression, Conversion Hysteria, Schizophrenia, and Social Introversion scales.

As with the other clinical conditions, the possible placebo effect of increased endorphins or dopamine related to increases in alpha and beta remains a possibility. Contrary to that possibility, Sokhadze and Daniels (2016) reported on changes in selfperception of positive emotional state following 12 sessions of NT involving increasing the prefrontal relative power of $35-45 \mathrm{~Hz}$ EEG band at Fpz (middle of forehead). The NT training resulted in a significant linear increase of the relative power of the $35-45 \mathrm{~Hz}$ gamma measure and increase in self-report of happiness scores. Follow-up at 3.9 months showed maintenance of gains in happiness measure as well as MicroCog and IVA+Plus neurocognitive tests. The foundation for the research was raised in three previous publications (Cowan, \& Albers, 2009; Cowan \& Sokhadze, 2010, 2011). In addition, there is no research that the author is aware of which ties increases in the gamma frequency to a placebo effect.

\section{Anxiety}

Walker (2009) studied 19 PTSD patients with alpha/theta NT and obtained significant reductions in anxiety, while the control group $(N=4)$ did not show any reductions in anxiety. A decline in alpha activity has been reported in anxiety disorders using the qEEG (Buchsbaum et al., 1985; Heller, Nitschke, Etienne, \& Miller, 1997).

The qEEG abnormalities in 100 anxious patients were reported by Gurnee (2003). He described six qEEG subtypes-high beta, high alpha, low alpha, cingulate dysfunction, high mean frequency beta, and high mean frequency alpha. Interventions directed towards these problems were generally effective in reducing anxiety. The placebo effect (alpha increase) could be employed to account for some of these improvements. However, the explanation of a sole alpha placebo effect across these diverse EEG 
conditions faces the same problem previously discussed of alpha increases related to general magnitude increases across the different frequencies.

\section{Cognitive - Memory}

Thornton and Carmody (2013) reported on significant improvements in cognitive function in a group of 79 participants, including normal individuals, traumatic brain-injured, and specific learning disabilities (children, adult). Table 1 presents the results of the 2013 article as well as two previous articles (Thornton \& Carmody, 2005, 2008).

\begin{tabular}{|c|c|c|c|c|}
\hline & $\begin{array}{l}\text { Auditory } \\
\text { Memory } \\
\text { SD Effect }\end{array}$ & $\begin{array}{l}\text { Auditory } \\
\% \text { Effect }\end{array}$ & $\begin{array}{l}\text { Reading } \\
\text { Memory } \\
\text { SD Effect }\end{array}$ & $\begin{array}{l}\text { Reading } \\
\% \text { Effect }\end{array}$ \\
\hline $\begin{array}{l}\text { Normal } \\
(n=12)\end{array}$ & 1.66 & $59 \%$ & 1.29 & $101 \%$ \\
\hline $\begin{array}{c}\text { TBI } \\
(N=36)\end{array}$ & $\begin{array}{c}2.3 \\
(N=36)\end{array}$ & & $\begin{array}{c}1.85 \\
(N=13)\end{array}$ & $143 \%$ \\
\hline $\begin{array}{c}\text { Adult SLD } \\
(N=17)\end{array}$ & 1.42 & $86 \%$ & 1.71 & $219 \%$ \\
\hline $\begin{array}{c}\text { Child SDL } \\
(n=14)\end{array}$ & 1.28 & $74 \%$ & 1.38 & $225 \%$ \\
\hline $\begin{array}{c}\text { Total } \\
\text { (average; } \\
N=79 \text { ) }\end{array}$ & 1.67 & $73 \%$ & 1.56 & $172 \%$ \\
\hline
\end{tabular}

The following definitions of the QEEG variables are as follows:

- RP: Relative Magnitude/Microvolt or Relative Power: the relative magnitude of a band defined as the absolute microvolt of the particular band divided by the total microvolt generated at a particular location across all bands

- M: Absolute Magnitude: the average absolute magnitude (as defined in microvolts) of a band over the entire epoch (one second)

- PA: Peak Amplitude: the peak amplitude of a band during an epoch (defined in microvolts)

- PF: Peak Frequency: the peak frequency of a band during an epoch (defined in frequency)

\section{Connectivity Measures}

- Spectral Correlation Coefficient (SCC): spectral morphology comparison correlation between two channels using the formula $(\Sigma|X||Y|)^{2} /\left(\Sigma|X|^{2}\right.$ $\left.\Sigma|Y|^{2}\right)$ expressed in percent, where $X$ and $Y$ represent the Fourier series of the two channels and $\sum$ represents the summation within a band's frequency range.
- Phase: peak amplitude phase difference between two channels using the formula $100\left(1-\left|\theta_{1}-\theta_{2}\right| / \pi\right)$. $A$ value of 100 percent represents zero degrees out of phase and a value of zero percent represents 180 degrees out of phase.

The bandwidths were grouped according to the following divisions: delta $(0-4 \mathrm{~Hz})$, theta $(4-8 \mathrm{~Hz})$, alpha $(8-13 \mathrm{~Hz})$, beta1 $(13-32 \mathrm{~Hz})$, beta2 $(32-64$ $\mathrm{Hz})$.

An analysis of the changes in the relative power values of the different frequencies showed that the relative power of beta2 $(32-64 \mathrm{~Hz})$ was the only frequency that showed significant improvements when analyzed using confidence intervals. The relative power of beta2 was a main focus of the interventions. Thus, a placebo's effect on the relative power value of beta2 seems unlikely, given the previous discussion, as the placebo response is focused on the alpha and lower beta frequencies.

The correlation between \% improvement in relative power of beta2 was most evident in the TBI group $(+.46)$ and adult specific learning disability $(+.77)$ in the auditory memory condition.

The conclusion for the coherence values was that "the average raw value of the Spectral Correlation Coefficient (SCC) change for alpha was 6.1 points (2.09 SD), for SCC beta1 $(13-32 \mathrm{~Hz}) 6.53$ points (1.81 SD), and for beta2 $(32-64 \mathrm{~Hz}) 7.5$ points $(1.77$ $S D) . "$

Thus, the NT interventions were able to obtain significant increases in the relative power of beta2 and coherence values from alpha to beta2, across all subjects.

As previously noted, the placebo's effect is in the alpha or beta frequency and not the gamma frequency. In addition, alpha values are generally negatively related to the gamma frequency values (Thornton, 2016). Thus, it appears implausible that the placebo effect could account for these changes as there is no evidence to support the effect of placebos on relative power of beta2 $(32-64 \mathrm{~Hz})$ or any changes in the coherence and phase values of the beta1 of beta2 frequencies, which are critical to successful cognitive functioning (Thornton, 2016). 


\section{Discussion / Conclusions}

Thibault and Raz (2017) make the argument that "placebo effects dominate EEG-nf outcomes. Whereas most neurofeedback experts acquiesce to this insight" (p. 684). The support for this statement resides in following quotes from experts:

It would be naïve to believe that neurofeedback offers an adequate and sufficient treatment for any disorder (Joel Lubar, personal communication, 2016)...."It would be foolish to conclude that a foundation of knowledge has been realized enabling textbooks to be written [on EEG- nf]" (Gruzelier, 2014, p. 178).... Niels Birbaumer proposed that the cumulative evidence in favor of EEG-nf is preliminary and we stand to benefit from more controlled evidence to confirm that genuine feedback is a necessary component to achieve positive treatment outcomes (personal communication, 2016). (p. 688)

These direct quotes do not mention placebo effects, so it is difficult to discern how the authors came to that conclusion.

Thibault and Raz (2017) further state that "in light of the comparable benefits of veritable-versus-sham feedback, conflicts of interest, and a weak theoretical underpinning, advocating for EEG-nf poses a conundrum...Sparse evidence supports the idea that humans can reliably modulate EEG-nf signals" ( $p$. 684). This conclusion is in stark contrast to the Thornton and Carmody (2013) and Thornton (2006) research study of cognitive changes, which consistently documented the ability of the approach to change the EEG signals along a number of measures and across different subjects.

A "weak theoretical underpinning" is a grossly inaccurate way to characterize operant conditioning, a concept Skinner proposed in 1938 (Skinner, 1938). The research cited in this article does show the effectiveness of NT over sham treatment, thus providing research support for its effectiveness which effectively addresses criticisms. In addition, the NT as a placebo argument has considerable problems as:

1) It claims "ubiquitous placebo influences" of NT (Thibault \& Raz, 2017). This statement is not consistent with research on placebo effects, which show only $7 \%$ overall effectiveness across different clinical conditions, only $32 \%$ of patients respond to placebos, a genetic predisposition, and a lower percentage of patient responsiveness than an effective drug (for pain).

2) The biochemical effect of the endorphins and dopamine levels of a placebo are not sufficient to explain the short-term and longterm effects, given their half-lives. However, this criticism requires qualification as some patients show the placebo effect long after the half-lives of the endorphins and dopamine have passed. A possible answer is that the patient is continuing to produce these neurotransmitters by continuing to affect the alpha frequency. If this is the case, then we are still left with the problem of the cause. Is the continuing alpha activity the result of a placebo effect or effective operant conditioning? Since both the biofeedback typical program addresses alpha and the biochemical effect appears to be in the alpha frequency, it appears that the data presently available is unable to directly address that question.

3) The relationship of the biochemical effects on the alpha and beta frequencies appear to possibly explain the NT results. However, the gamma responsivity is inconsistent with the biochemical hypothesis as there is no evidence of a biochemical effect on this frequency.

4) Occam's razor states that among competing hypotheses, the one with the fewest assumptions should be selected. NT as a placebo has considerable problems in its explanation of the research findings. It is simpler to assume and more consistent with the literature that operant conditioning is the effective operant rather than some inconsistent and intermittent placebo effect.

5) This article has presented empirical evidence that humans can have an effect on the EEG signal. The relationship between the qEEG variables and cognitive performance is a complex one. It is difficult to assert that the placebo's effect is on all/most of the variables that relate to performance. If we reward a qEEG variable that has an empirical relationship to performance (memory) and the variable and memory performance improve, it is logical to conclude that the 
intervention is not a placebo. To assert that a placebo knows what $q E E G$ variable relates to performance and increases the values of those specific variables (among the 2,000+ available) is a difficult position to defend.

In conclusion, the effect of a placebo or subject's expectation can be linked to biochemical and electrophysiological activity. However, the relationship is a complex one and the ability of a placebo to explain all the effects is not credible, given the evidence reviewed. The alternate explanation that operant conditioning is causing the changes in the EEG signals is a more plausible and simpler one.

\section{References}

Amanzio, M., \& Benedetti, F. (1999). Neuropharmacological dissection of placebo analgesia: Expectation-activated opioid systems versus conditioning-activated specific subsystems. The Journal of Neuroscience, 19(1), 484-494. http://dx.doi.org/10.1523/JNEUROSCI.19-01-00484.1999

Arruda, J. E., Zhang, H., Amoss, R. T., Coburn, K. L., \& Aue, W. R. (2009). Rhythmic oscillations in quantitative EEG measured during a continuous performance task. Applied Psychophysiology and Biofeedback, 34(1), 7-16. http://dx.doi.org/10.1007/s10484-008-9071-0

Auckland District Health Board (ADHB). (2018). Newborn Services Drug Protocol. Dopamine hydrochloride. Retrieved from http://www.adhb.govt.nz/newborn/DrugProtocols /DopaminePharmacology.htm

Banich, M. T., Milham, M. P., Atchley, R., Cohen, N. J., Webb, A., Wszalek, T., ... Magin, R. (2000). fMRI studies of Stroop tasks reveal unique roles of anterior and posterior brain systems in attentional selection. Journal of Cognitive Neuroscience, 12(6), 988-1000. http://dx.doi.org/10.1162 /08989290051137521

Barkley, R. A. (1998). Attention-deficit hyperactivity disorder: A handbook for diagnosis and treatment (2nd ed.). New York, NY: Guilford Press.

Barry, R. J., Clarke, A. R., \& Johnstone, S. J. (2003). A review of electrophysiology in attention-deficit/hyperactivity disorder: I. Qualitative and quantitative electroencephalography. Clinical Neurophysiology, 114(2), 171-183. http://dx.doi.org/10.1016 IS1388-2457(02)00362-0

Beecher, H. K. (1955). The powerful placebo. Journal of the American Medical Association, 159(17), 1602-1606. http://dx.doi.org/10.1001/jama.1955.02960340022006

Benedetti, F., Colloca, L., Torre, E., Lanotte, M., Melcarne, A., Pesare, M., ... Lopiano, L. (2004). Placebo-responsive Parkinson patients show decreased activity in single neurons of subthalamic nucleus. Nature Neuroscience, 7(6), 587-588. http://dx.doi.org/10.1038/nn1250

Bresnahan, S. M., Barry, R. J., Clarke, A. R., \& Johnstone, S. J. (2006). Quantitative EEG analysis in dexamphetamineresponsive adults with attention-deficit/hyperactivity disorder. Psychiatry Research, 141(2), 151-159. http://dx.doi.org /10.1016/j.psychres.2005.09.002

Buchsbaum, M. S., Hazlett, E., Sicotte, N., Stein, M., Wu, J., \& Zetin, M. (1985). Topographic EEG changes with benzodiazepine administration in generalized anxiety disorder. Biological Psychiatry, 20(8), 832-842. http://dx.doi.org/10.1016/0006-3223(85)90208-2

Bush, G., Frazier, J. A., Rauch, S. L., Seidman, L. J., Whalen, P. J., Jenike, M. A., ... Biederman, J. (1999). Anterior cingulate cortex dysfunction in attention-deficit/hyperactivity disorder revealed by $\mathrm{fMRI}$ and the Counting Stroop, Biological Psychiatry, 45(12), 1542-1552.

Chabot, R. J., \& Serfontein, G., (1996). Quantitative electroencephalographic profiles of children with attention deficit disorder. Biological Psychiatry, 40(10), 951-963. http://dx.doi.org/10.1016/0006-3223(95)00576-5

Cheon, E.-J., Koo, B.-H., \& Choi, J.-H. (2016). The efficacy of neurofeedback in patients with major depressive disorder: An open labeled prospective study. Applied Psychophysiology and Biofeedback, 41(1), 103-110. http://dx.doi.org/10.1007 /s10484-015-9315-8

Cherry, K. (2018, November 14). How the Placebo Effect Works in Psychology. Retrieved from https://www.verywellmind.com /what-is-the-placebo-effect-2795466

Clarke, A. R., Barry, R. J., McCarthy, R., Selikowitz, M., \& Johnstone, S. J. (2007). Effects of stimulant medications on the EEG of girls with attention-deficit/hyperactivity disorder. Clinical Neurophysiology, 118(12), 2700-2708. http://dx.doi.org/10.1016/j.clinph.2007.08.020

Cowan, J. D., \& Albers, S. A. (2009). Manual for the peak brain happiness trainer: Exited happiness and Neureka! Protocols. Goshen, KY: Peak Achievement Training.

Cowan, J. D., \& Sokhadze, E. (2011). Prefrontal gamma neurofeedback improves emotional state and cognitive function. Applied Psychophysiology and Biofeedback, 36, S220.

Cowan, J., \& Sokhadze, E. (2010, September 29-October 3). Happiness specifically increases a clarified $40 \mathrm{~Hz}$ EEG rhythm used for neurofeedback. Presented at the 18th International Society Neurofeedback Research Annual Conference, Denver, CO

Crabbe, J. B., \& Dishman, R. K. (2004). Brain electrocortical activity during and after exercise: A quantitative synthesis Psychophysiology, 41(4), 563-574. http://dx.doi.org/10.1111 /j.1469-8986.2004.00176.x

Dalayeun, J. F., Norès, J. M., Bergal, S. (1993). Physiology of betaendorphins. A close-up view and a review of the literature. Biomedicine \& Pharmacotherapy, 47(8), 311-320. http://dx.doi.org/10.1016/0753-3322(93)90080-5

de la Fuente-Fernández, R., Ruth, T. J., Sossi, V., Schulzer, M., Calne, D. B., \& Stoessl, A. J. (2001). Expectation and dopamine release: Mechanism of the placebo effect in Parkinson's disease. Science, 293(5532), 1164-1166. http://dx.doi.org/10.1126/science.1060937

Eippert, F., Bingel, U., Schoell, E. D., Yacubian, J., Klinger, R., Lorenz, J., \& Büchel, C. (2009). Activation of the opioidergic descending pain control system underlies placebo analgesia. Neuron, 63(4), 533-543. http://dx.doi.org/10.1016 /j.neuron.2009.07.014

Ernst, M., Zametkin, A. J., Matochik, J. A., Liebenauer, L. Fitzgerald, G. A., \& Cohen, R. M. (1994). Effects of intravenous dextroamphetamine on brain metabolism in adults with attention-deficit/hyperactivity disorder (ADHD): Preliminary findings. Psychopharmacology Bulletin, 30(20), 219-225.

Feinberg, C. (2013, January-February). The placebo phenomenon. An ingenious researcher finds the real ingredients of "fake" medicine. Harvard Magazine, 115(3), 3639 .

Foley, K. M., Kourides, I. A., Inturrisi, C. E., Kaiko, R. F., Zaroulis, C. G., Posner, J. B., ... Li, C. H. (1979). $\beta$-Endorphin: Analgesic and hormonal effects in humans. Proceedings of the National Academy of Sciences of the United States of America, 76(10), 5377-5381.

Furmark, T., Appel, L., Henningsson, S., Åns, F., Faria, V., Linnman, C., ... Fredrikson, M. (2008). A link between serotonin-related gene polymorphisms, amygdala activity, and placebo-induced relief from social anxiety. The Journal of Neuroscience, 28(49), 13066-13074. http://dx.doi.org /10.1523/JNEUROSCI.2534-08.2008 
Gabrielli, W. F., Mednick, S. A., Volavka, J., Pollock, V. E., Schulsinger, F. \& Itil, T. M. (1982). Electroencephalograms in children of alcoholic fathers. Psychophysiology, 19(4), 404407.

Gani, C., Birbaumer, N., \& Strehl, U. (2008). Long term effects after feedback of slow cortical potentials and of theta-betaamplitudes in children with attention deficit/hyperactivity disorder (ADHD). International Journal of Bioelectromagnetics, 10(4), 209-232.

Gevensleben, H., Holl, B., Albrecht, B., Schlamp, D., Kratz, O., Studer, P., ... Heinrich, H. (2009). Distinct EEG effects related to neurofeedback training in children with ADHD: A randomized controlled trial. International Journal of Psychophysiology, 74(2), 149-157. http://dx.doi.org/10.1016 /j.ijpsycho.2009.08.005

Gevensleben, H., Holl, B., Albrecht, B., Schlamp, D., Kratz, O., Studer, P., ... Heinrich, H. (2010). Neurofeedback training in children with ADHD: 6-month follow-up of a randomised controlled trial. European Child \& Adolescent Psychiatry, 19(9), 715-724. http://dx.doi.org/10.1007/s00787-010-0109-5

Gevensleben, H., Holl, B., Albrecht, B., Vogel, C., Schlamp, D., Kratz, O., ... Heinrich, H. (2009). Is neurofeedback an efficacious treatment for ADHD? A randomized controlled clinical trial. Journal of Child Psychology and Psychiatry, 50(7), 780-789. http://dx.doi.org/10.1111/j.1469-7610.2008.02033.x

Ghaziri, J., Tucholka, A., Larue, V., Blanchette-Sylvestre, M., Reyburn, G., Gilbert, G., ... Beauregard, M. (2013). Neurofeedback training induces changes in white and gray matter. Clinical EEG and Neuroscience, 44(4), 265-272. http://dx.doi.org/10.1177/1550059413476031

Gracely, R. H., Dubner, R., Wolskee, P. J., \& Deeter, W. R. (1983). Placebo and naloxone can alter post-surgical pain by separate mechanisms. Nature, 306(5940), 264-265. http://dx.doi.org/10.1038/306264a0

Grevert, P., Albert, L. H., \& Goldstein, A. (1983). Partial antagonism of placebo analgesia by naloxone. Pain, 16(1), 129-143. http://dx.doi.org/10.1016/0304-3959(83)90203-8

Grin-Yatsenko, V. A., Othmer, S., Ponomarev, V. A., Evdokimov, S. A., Konoplev, Y. Y., \& Kropotov, J. D. (2018) Infra-low frequency neurofeedback in depression: Three case studies. NeuroRegulation, 5(1), 30-42. http://dx.doi.org /10.15540/nr.5.1.30

Gruzelier, J. H. (2014). EEG-neurofeedback for optimising performance. III: A review of methodological and theoretical considerations. Neuroscience \& Biobehavioral Reviews, 44, 159 -182. http://dx.doi.org/10.1016/j.neubiorev.2014.03.015

Gunkelman, J. (2009, December 23). Drug exposure and EEG/qEEG findings. Retrieved from http://brainm.com /software/pubs/Gunkelman Drug exposure and EEG.pdf

Gurnee, R. (2003). QEEG subtypes of anxiety. Presented at the International Society for Neurofeedback and Research 11th Annual Conference, Houston, TX.

Hall, K. T., Lembo. A. J., Kirsch. I., Ziogas, D. C., Douaiher, J., Jensen, K. B., ... Kaptchuk, T. J. (2012). Catechol-Omethyltransferase val158met polymorphism predicts placebo effect in irritable bowel syndrome. PLOS ONE, 7(10), e48135. http://dx.doi.org/10.1371/journal.pone.0048135

Hall, K. T., Loscalzo, J., \& Kaptchuk, T. J. (2015). Genetics and the placebo effect: The placebome. Trends in Molecular Medicine, 21(5), 285-294. http://dx.doi.org/10.1016 /j.molmed.2015.02.009

Hamilton, L. S., Levitt, J. G., O'Neil, J., Alger, J. R., Luders, E., Phillips, O. R., ... Narr, K. L. (2008). Reduced white matter integrity in attention-deficit hyperactivity disorder. Neuroreport, 19(17), $\quad$ 1705-1708. http://dx.doi.org/10.1097 IWNR.0b013e3283174415

Hammond, D. C. (2005). Neurofeedback with anxiety and affective disorders. Child and Adolescent Psychiatric Clinics of North
America, 14(1), 105-123. http://dx.doi.org/10.1016 /j.chc.2004.07.008

Heller, W., Nitschke, J. B., Etienne, M. A., \& Miller, G. A. (1997). Patterns of regional brain activity differentiate types of anxiety. Journal of Abnormal Psychology, 106(3), 376-385. http://dx.doi.org/10.1037/0021-843X.106.3.376

Hróbjartsson, A., \& Gøtzsche, P. A. (2010). Placebo interventions for all clinical conditions. Cochrane Database of Systematic Reviews, $1.1 \quad \mathrm{http} / / / \mathrm{dx}$.doi.org/10.1002 /14651858.CD003974.pub3

Kaptchuk, T. J., Friedlander, E., Kelley, J. M., Sanchez, M. N., Kokkotou, E., Singer, J. P., ... Lembo, A. J. (2010). Placebos without deception: A randomized controlled trial in irritable bowel syndrome. PLoS ONE, 5(12), e15591. http://dx.doi.org /10.1371/journal.pone.0015591

Kong, J., Gollub, R. L., Rosman, I. S., Webb, J. M., Vangel, M. G., Kirsch, I., \& Kaptchuk, T. J. (2006). Brain activity associated with expectancy-enhanced placebo analgesia as measured by functional magnetic resonance imaging. The Journal of Neuroscience, 26(2), 381-388. http://dx.doi.org/10.1523 /JNEUROSCI.3556-05.2006

Konrad, A., Dielentheis, T. F., Masri, D. E., Dellani, P. R., Stoeter, P., Vucurevic, G., \& Winterer, G. (2012). White matter abnormalities and their impact on attentional performance in adult attention-deficit/hyperactivity disorder. European Archives of Psychiatry and Clinical Neuroscience, 262(4), 351360. http://dx.doi.org/10.1007/s00406-011-0251-1

Leins, U., Goth, G., Hinterberger, T., Klinger, C., Rumpf, N., \& Strehl, U. (2007). Neurofeedback for children with ADHD: A comparison of SCP and theta-beta protocols. Applied Psychophysiology and Biofeedback, 32(2), 73-88. http://dx.doi.org/10.1007/s10484-007-9031-0

Leuchter, A. F., Cook, I. A., Witte, E. A., Morgan, M., \& Abrams, M. (2002). Changes in brain function of depressed subjects during treatment with placebo. The American Journal of Psychiatry, 159(1), 122-129. http://dx.doi.org/10.1176 /appi.ajp.159.1.122

Lévesque, J., Beauregard, M., \& Mensour, B. (2006). Effect of neurofeedback training on the neural substrates of selective attention in children with attention-deficit/hyperactivity disorder: A functional magnetic resonance imaging study. Neuroscience Letters, 394(3), 216-221. http://dx.doi.org /10.1016/j.neulet.2005.10.100

Levine, J. D., Gordon, N. C., Jones, R. T., \& Fields, H. L. (1978). The narcotic antagonist naloxone enhances clinical pain. Nature, 272, 826-827.

Lipman, J. J., Miller, B. E., Mays, K. S., Miller, M. N., North, W. C., \& Byrne, W. L. (1990). Peak B endorphin concentration in cerebrospinal fluid: reduced in chronic pain patients and increased during the placebo response. Psychopharmacology, 102(1), 112-116. http://dx.doi.org /10.1007/BF02245754

Lubar, J. F., Swartwood, M. O., Swartwood, J. N., \& Timmermann, D. L. (1996). Quantitative EEG and auditory event-related potentials in the evaluation of attention-deficit/hyperactivity disorder: Effects of methylphenidate and implications for neurofeedback training. [Monograph: Assessment of AttentionDeficit/Hyperactivity Disorders]. Journal of Psychoeducational Assessment, 143-204.

Matochik, J. A., Liebenauer, L. L., King, A. C., Szymanski, H. V., Cohen, R. M., \& Zametkin, A. J. (1994). Cerebral glucose metabolism in adults with attention deficit hyperactivity disorder after chronic stimulant treatment. American Journal of Psychiatry, 151(5), 658-664. http://dx.doi.org/10.1176 lajp.151.5.658

Matsuura, M., Okubo, Y., Toru, M., Kojima, T., He, Y., Hou, Y., ... Lee, C. K. (1993). A cross-national EEG study of children with emotional and behavioral problems: A WHO collaborative study in the Western Pacific Region. Biological Psychiatry, 34(1-2), 59-65. http://dx.doi.org/10.1016/00063223(93)90257-E 
Mimasa, F., Hayashi, T., Shibata, M., Yoshitake, Y., Nishijima, Y., \& Moritani, T. (1996). Movement of electroroencephalogram and plasm $\beta$-endorphin in the aerobic exercise. Japanese Journal of Physical Fitness and Sports Medicine, 45(5), 519526. http://dx.doi.org/10.7600/jspfsm1949.45.519

Molteni, E., Bianchi, A. M., Butti, M., Reni, G., \& Zucca, C., (2007). Analysis of the dynamical behaviour of the EEG rhythms during a test of sustained attention. Conference Proceedings: Annual International Conference of the IEEE Engineering in Medicine and Biology Society, 1298-1301. http://dx.doi.org/10.1109/IEMBS.2007.4352535

Monastra, V. J., Monastra, D. M., \& George, S. (2002). The effects of stimulant therapy, EEG biofeedback, and parenting style on the primary symptoms of attention-deficit/hyperactivity disorder. Applied Psychophysiology and Biofeedback, 27(4), 231-249. http://dx.doi.org/10.1023 /A:1021018700609

Nestler, E. J., Hyman, S. E., \& Malenka, R. C., (2009). Molecular Neuropharmacology: A Foundation for Clinical Neuroscience (2nd ed., p. 321). New York, NY: McGraw-Hill Medical.

Niogi, S., Mukherjee, P., Ghajar, J., \& McCandliss, B. D. (2010). Individual differences in distinct components of attention are linked to anatomical variations in distinct white matter tracts. Frontiers in Neuroanatomy, 4, 2. http://dx.doi.org/10.3389 Ineuro.05.002.2010

Pavuluri, M. N., Yang, S., Kamineni, K., Passarotti, A. M., Srinivasan, G., Harral, E. M., ... Zhou, X. J. (2009). Diffusion tensor imaging study of white matter fiber tracts in pediatric bipolar disorder and attention-deficit/hyperactivity disorder. Biological Psychiatry, 65(7), 586-593. http://dx.doi.org /10.1016/j.biopsych.2008.10.015

Peniston, E. G., \& Kulkosky, P. J. (1989). Alpha-theta brainwave training and beta-endorphin levels in alcoholics. Alcoholism, Clinical \& Experimental Research, 13(2), 271-279. http://dx.doi.org/10.1111/j.1530-0277.1989.tb00325.x

Petrovic, P., Dietrich, T., Fransson, P., Andersson, J., Carlsson, K., \& Ingvar, M. (2005). Placebo in emotional processinginduced expectations of anxiety relief activate a generalized modulatory network. Neuron, 46(6), 957-969. http://dx.doi.org/10.1016/j.neuron.2005.05.023

Petrovic, P., Kalso, E., Petersson, K. M., \& Ingvar, M. (2002). Placebo and opioid analgesia-imaging a shared neuronal network. Science, 295(5560), 1737-1740. http://dx.doi.org /10.1126/science.1067176

Pfefferbaum, A., Berger, P. A., Elliot, G. R., Tinklenberg, J. R., Kopell, B. S., Barchas, J. D., \& Li, C. H. (1979). Human EEG response to beta-endorphin. Psychiatry Research, 1(1), 8388. http://dx.doi.org/10.1016/0165-1781(79)90031-3

Qiu, M.-G., Ye, Z., Li, Q.-Y., Liu, G.-J., Xie, B., \& Wang, J. (2011). Changes of brain structure and function in ADHD children. Brain Topography, 24(3-4), 243-252. http://dx.doi.org /10.1007/s10548-010-0168-4

Raymond, J., Varney, C., Parkinson, L. A., \& Gruzelier, J. H. (2005). The effects of alpha/theta neurofeedback on personality and mood. Cognitive Brain Research, 23(2-3), 287-292. http://dx.doi.org/10.1016/j.cogbrainres.2004.10.023

Rossi, E. L. (1986). The psychobiology of mind-body healing: New concepts of therapeutic hypnosis (Rev. ed.). New York, NY: W. W. Norton \& Company, Inc.

Saletu, B., Anderer, P., Saletu-Zyhlarz, G. M., Arnold, O., \& Pascual-Marqui, R. D. (2002). Classification and evaluation of the pharmacodynamics of psychotropic drugs by single-lead pharmaco-EEG, EEG mapping and tomography (LORETA). Methods and Findings in Experimental and Clinical Pharmacology, 24(Suppl. C), 97-120.

Scott, D. J., Stohler, C. S., Egnatuk, C. M., Wang, H., Koeppe, R. A., \& Zubieta, J.-K. (2008). Placebo and nocebo effects are defined by opposite opioid and dopaminergic responses. Archives of General Psychiatry, 65(2), 220-231. http://dx.doi.org/10.1001 /archgenpsychiatry.2007.34
Scott, W. C., Kaiser, D., Othmer, S., \& Sideroff, S. I. (2005). Effects of an EEG biofeedback protocol on a mixed substance abusing population. The American Journal of Drug and Alcohol Abuse, 31(3), 455-469. http://dx.doi.org/10.1081 /ADA-200056807

Sherlin, L., Arns, M. Lubar, J., \& Sokhadze, E. (2010). A position paper on neurofeedback for treatment of ADHD. Journal of Neurotherapy, 14(2), 66-78. http://dx.doi.org/10.1080 /10874201003773880

Skinner, B. F. (1938). The behavior of organisms: An experimental analysis. New York, NY: Appleton-Century-Crofts, Inc.

Snyder, S. M., \& Hall, J. R. (2006). A meta-analysis of quantitative EEG power associated with attention-deficit/hyperactivity disorder. Journal of Clinical Neurophysiology, 23(5), 441-456. http://dx.doi.org/10.1097/01.wnp.0000221363.12503.78

Sokhadze, E. M., \& Daniels, R. (2016). Effects of prefrontal $40 \mathrm{~Hz}-$ centered EEG band neurofeedback on emotional state and cognitive functions in adolescents. Adolescent Psychiatry, 6(2), 116-129. http://dx.doi.org/10.2174 /2210676606666161025115616

Sokhadze, T. M., Cannon, R. L., \& Trudeau, D. L. (2008). EEG biofeedback as a treatment for substance use disorders: Review, rating of efficacy, and recommendations for further research. Applied Psychophysiology and Biofeedback, 33(1), 1-28. http://dx.doi.org/10.1007/s10484-007-9047-5

Staddon, J. E. R., \& Cerutti, D. T. (2003). Operant conditioning. Annual Review of Psychology, 54, 115-144. http://dx.doi.org/10.1146/annurev.psych.54.101601.145124

Thibault, R. T., \& Raz, A. (2017). The psychology of neurofeedback: Clinical intervention even if applied placebo. American Psychologist, 72(7), 679-688. http://dx.doi.org /10.1037/amp0000118

Thornton, K. E. (2006). No child left behind goals (and more) are attainable with neurocognitive interventions (Vol. 1). North Charleston, SC: Booksurge Press.

Thornton, K. (2016). How the cognitive brain works: The quantitative EEG and cognition. Clover, SC: Kirtley Thornton. Printed by Create Space.

Thornton, K. E., \& Carmody, D. P. (2005). EEG biofeedback for reading disabilities and traumatic brain injuries. Child and Adolescent Psychiatric Clinics of North America, 14(1), 137162.

Thornton, K. E., \& Carmody, D. P. (2008). Efficacy of traumatic brain injury rehabilitation: interventions of qEEG-guided biofeedback, computers, strategies, and medications. Applied Psychophysiology and Biofeedback, 33(2), 101-124. http://dx.doi.org/10.1007/s10484-008-9056-z

Thornton, K. E., \& Carmody, D. P. (2013). The relation between memory improvement and qEEG changes in three clinical groups as a result of EEG biofeedback treatment. Journal of Neurotherapy, 17(2), 116-132. http://dx.doi.org/10.1080 /10874208.2013.785183

van Ewijk, H., Heslenfeld, D. J., Zwiers, M. P., Buitelaar, J. K., \& Oosterlaan, J. (2012). Diffusion tensor imaging in attention deficit/hyperactivity disorder: A systematic review and metaanalysis. Neuroscience \& Biobehavioral Reviews, 36(4), 10931106. http://dx.doi.org/10.1016/j.neubiorev.2012.01.003

Volavka, J., James, B., Reker, D., \& Cho, D. (1979). Electroencephalographic and other effects of naloxone in normal men. Life Sciences, 25(14), 1267-1272. http://dx.doi.org/10.1016/0024-3205(79)90471-5

Wager, T. D., Rilling, J. K., Smith, E. E., Sokolik, A., Casey, K. L., Davidson, R. J., ... Cohen, J. D. (2004). Placebo-induced changes in $\mathrm{fMRI}$ in the anticipation and experience of pain. Science, 303(5661), 1162-1167. http://dx.doi.org/10.1126 /science.1093065

Wager, T. D., Scott, D. J., \& Zubieta, J. K. (2007). Placebo effects on human mu-opioid activity during pain. Proceedings of the National Academy of Sciences of the United States of America, 
104(26), 11056-11061. http://dx.doi.org/10.1073 /pnas.0702413104

Walker, J. E. (2009). Anxiety associated with post traumatic stress disorder-The role of quantitative electroencephalograph in diagnosis and in guiding neurofeedback training to remediate the anxiety. Biofeedback, 37(2), 67-70. http://dx.doi.org/10.5298/1081-5937-37.2.67

Wang, S.-Y., Lin, I.-M., Peper, E., Chen, Y.-T., Yeh, Y.-C., ... Chu, C. (2016). The efficacy of neurofeedback among patients with major depressive disorder: Preliminary study. NeuroRegulation, 3(3), 127-134. http://dx.doi.org/10.15540 Inr.3.3.127

Wechsler, M. E., Kelley, J. M., \& Kaptchuk, T. J. (2011). Placebos and other interventions in asthma. The New England Journal of Medicine, 365(15), 1447.

World Research Foundation (WRF) Staff. (n.d.) The power of mind and the promise of placebo. Retrieved from http://www.wrf.org/alternative-therapies/power-of-mindplacebo.php

Zubieta, J.-K., Bueller, J. A., Jackson, L. R., Scott, D. J., Xu, Y., Koeppe, R. A., ... Stohler, C. S. (2005). Placebo effects mediated by endogenous opioid activity on mu-opioid receptors. The Journal of Neuroscience, 25(34), 7754-7762. http://dx.doi.org/10.1523/JNEUROSCI.0439-05.2005

Zubieta, J.-K., \& Stohler, C. S. (2009). Neurobiological mechanisms of placebo responses. Annals of the New York Academy of Sciences, 1156(1), 198-210. http://dx.doi.org /10.1111/j.1749-6632.2009.04424.x

Received: August 10, 2018

Accepted: November 26, 2018

Published: December 7, 2018 